\title{
Factores de riesgo psicosocial intralaborales y su relación con dolor músculo esquelético en docentes universitarios
}

\author{
Psychosocial and intra-labor risk factors and their relationship with skeletal muscle pain in university professors
}

María Alexandra Gutiérrez-Calderón ${ }^{*}$ orcid.org/0000-0002-7576-5273

Kelly Mercedes Diaz-Therán' orcid.org/0000-0001-5893-1439

1. Corporación Universitaria del Caribe CECAR, Sincelejo. Sucre, Colombia.

Gutiérrez-Calderón MA, Díaz-Therán KM. Factores de riesgo psicosocial intralaboral y su relación con dolor músculo esquelético en docentes universitarios. Univ. Salud. Salud. Suplemento1: Especial Psicología y Trabajo. 2021; 23(3):329-336. DOI: https://doi.org/10.22267/rus.212303.247

\section{Resumen}

Introducción: Una de las primeras condiciones para la presencia de enfermedades músculo esqueléticas es la sintomatología dolorosa a nivel osteomuscular, siendo la causa más frecuente que acrecienta el riesgo de alteraciones ocupacionales. Objetivo: Identificar presencia de factores de riesgo psicosociales intralaborales y su relación con el dolor músculoesquelético en docentes. Materiales y métodos: Enfoque cuantitativo, descriptivo, con diseño transaccional, aplicado a 83 docentes universitarios de la ciudad Sincelejo (Colombia), con aplicación del cuestionario Nórdico Kourinka y la Batería para medir riesgo psicosocial propuesta por el Ministerio de Salud y de Protección Social. El procesamiento de la información se realizó por medio del SPSS 25 y el análisis de datos se efectuó a través de medidas de tendencia y pruebas de asociación. Resultados: Se encontró sintomatología dolorosa asociada a enfermedades músculo esqueléticas, con prevalencia en cuello, columna dorsal o lumbar, con significancia estadística a nivel de la asociación entre la variable dolor y factor de riesgo psicosocial intralaboral en el dominio control en el trabajo ( $p>0,05$ ). Conclusión: Es importante el reporte oportuno de datos fiables para evaluación y control de la sintomatología dolorosa y su relación con los factores internos de la carga laboral.

Palabras clave: Factores de riesgo; impacto psicosocial; enfermedades musculoesqueléticas. (Fuente: DeCS, Bireme).

\begin{abstract}
Introduction: One of the first conditions for the appearance of skeletal muscle diseases is osteomuscular pain, which is the most frequent factor that increases the risk for occupational illnesses. Objective: To identify the presence of intra-labor psychological factors and their relationship with musculoskeletal pain in professors. Materials and methods: A quantitative descriptive approach with a transactional design was applied with 83 university professors from the city of Sincelejo (Colombia). The Nordic Kourinka questionnaire and the Battery to measure psychosocial risk proposed by the Health and Social Protection Ministry were used. Information was processed with the SPSS 25 software, and data analysis was carried out through trend measures and association tests. Results: Symptoms of pain associated with musculoskeletal diseases were found, with prevalence of neck, dorsal or lumbar column pain. Also, there was a statistically significant difference between the pain variable and psychosocial intra-labor risk, in its work control domain $(p>0.05)$. Conclusion: The timely report of reliable data is important for the assessment and control of pain symptoms and their relationship with labor load internal factors.
\end{abstract}

Keywords: Risk Factors; psychosocial impact; musculoskeletal diseases. (Source: DeCS, Bireme).

\footnotetext{
*Autor de correspondencia

María Alexandra Gutiérrez-Calderón

e-mail: maria.gutierrezc@cecar.edu.co
} 


\section{Introducción}

Las alteraciones músculo esqueléticas son consideradas disfunciones, las cuales se caracterizan por una condición anómala de huesos, cápsulas articulares, estructuras tenoligamentosas y nerviosas que tienen como mecanismo causal una modificación a nivel neurosensitivo(1), generando así enfermedades que en la actualidad se consideran importantes para la salud del trabajador en países desarrollados o en aquellos en pro de desarrollo; presentándose en toda labor que en su progreso incluyan cargas dinámicas o estáticas sin el manejo correcto o sin las estrategias pertinentes para la disminución del riesgo(2,3).

En el sector de la educación, la mayoría de los docentes desconocen las alteraciones que pueden adquirir por la exposición continua a riesgos y peligros laborales durante su jornada de trabajo, teniendo en cuenta que su labor requiere la combinación de carga física estática de pie, sentado y en algunas ocasiones carga física mixta, además están expuestos diariamente a factores de riesgo psicológicos y sociales intralaborales(4).

Por lo anterior, es importante definir los factores de riesgo psicosociales como las características organizacionales vinculadas a los ambientes y contextos laborales que repercuten en los trabajadores, afectando de esta forma su bienestar laboral(5). Así también, estos factores de riesgo suponen las situaciones laborales aumentando la probabilidad de afectar la salud desde la integralidad, a través de unos mecanismos psicofisiológicos también conocidos como estrés(6).

Asimismo, se debe tener en cuenta factores fenómenos como la globalización, la competitividad, la vinculación contractual, así como la seguridad y sostenibilidad en el puesto de trabajo, que generan un detrimento en las condiciones laborales; creando situaciones en el trabajador que producen una serie de preocupaciones e inseguridades que de alguna manera ponen en peligro la salud(5). El sistema osteomuscular es de gran importancia en el desarrollo de las actividades del trabajo, sin embargo, la mayoría de los trabajadores no prestan atención a su nivel de salud y específicamente de actividad física necesaria para lograr tener una musculatura preparada que les permita mantener una postura estática y en muchos casos repetitiva, lo cual pone en jaque la armonía postural, y que algunas veces por comodidad tiende a convertirse en el uso de posturas inadecuadas, que genera una discordancia entre la capacidad física del cuerpo versus los requisitos físicos de la tarea(7,8).

Estas posiciones inadecuadas sostenidas por mucho tiempo son la causa de una carga agresiva en el sistema muscular y articular, como consecuencia de actividades laborales mal ejecutadas, algunas de estas actividades de desarrollo profesional son las relacionadas con acciones de fuerza, movimientos repetitivos con intensidad, frecuencia y duración definidos( $(9)$.

Todo este fenómeno postural, que incluye el movimiento con un rango de importancia para su ejecución, demuestra la necesidad de mantener un nivel de actividad física dentro de los parámetros establecidos por la Organización Mundial de la Salud (OMS), con el fin de disminuir los riesgos suscitados de la inactividad física que no sólo traen alteraciones a nivel del sistema musculoesquelético, si no también aumentan la probabilidad de enfermedades relacionadas con la obesidad, la diabetes, trastornos metabólicos, cardiacos entre otros(10), convirtiendo a la actividad física como la mejor estrategia de apoyo dinamizada a nivel laboral(11).

Así también es importante tener en cuenta que en la actualidad las instituciones de educación, a raíz de la emergencia sanitaria que activó la pandemia por el virus SARS-CoV-2 (COVID-19), como medida de seguridad se ha visto obligada a enviar a sus docentes a trabajar desde sus casas, donde no se encuentran las condiciones ergonómicas adecuadas para atender el trabajo que deben desempeñar; incrementando además la utilización de los dispositivos móviles, acomodación de espacios en la vivienda, mesas, sillas y elementos necesarios para el desarrollo adecuado de su labor y esto en gran medida aumenta la aparición de riesgos de tipo ergonómico, físico y psicosocial intra y extra laboral(11,12).

Lo anterior exige a las organizaciones abordar esta problemática desde una perspectiva multifactorial y multifocal, de aquí la importancia de la teoría basada en evidencia, donde algunos estudios reportan que los trastornos osteomusculares se consideran la enfermedad del mundo actual, que depende de múltiples causas y su manejo debe ser interdisciplinar por unas parte en la búsqueda de la recuperación de aquellas personas que la padecen y por otra, en la disminución significativa del riesgo en 
los actuales lugares de trabajo; de manera que se garantice la máxima comodidad de los individuos en sus puestos de trabajo y la armonía entre el sistema hombre- máquina-sistema de trabajo- ambiente(12). La discontinuidad de esta armonía en la cinética corporal puede llegar a producir perjuicios en la salud, entre ellos, las enfermedades musculo esqueléticas (EME)(13).

La mayoría de las EME que se originan como consecuencia del trabajo, se despliegan con el tiempo y son inducidas por el tipo de ocupación o por el contexto en el cual se desarrollan, además estas EME son catalogadas como una de las causas principales causas de ausentismo e incapacidad laboral $(4,14)$. Dentro de los trastornos del sistema musculo esquelético relacionados con el trabajo, se incluyen modificaciones en músculos, articulaciones, tendones, atrapamientos nerviosos y alteraciones neuro vasculares(15).

De esta manera, la Organización Mundial de la Salud (OMS) y la Organización Panamericana de la Salud (OPS), en pro de contribuir con el fortalecimiento de los medios de prevención de las EME consideradas profesionales, en cumplimiento con los designios recomendados por la Organización internacional del Trabajo (OIT) en 2018, proponen a los estados construir y aplicar encuestas nacionales que permitan levantar los perfiles de riesgos y peligros; definiendo las características de los procesos de trabajo(14) que permitan la realización de una matriz de exposición al riesgo, robustecer la aplicabilidad de la ergonomía, la higiene, seguridad y medicina ocupacional por medio de la organización de redes a nivel nacional, regional y local con especialistas en seguridad y salud en el trabajo(15). Todo lo anterior crea la necesidad de identificar los factores de riesgo psicosocial intralaborales y su relación con el dolor músculo esquelético en diferentes puestos de trabajo y para fines del presente artículo se realiza con docentes universitarios.

\section{Materiales y métodos}

\section{Tipo de estudio}

Este proceso se llevó a cabo desde una mirada positivista, con un enfoque cuantitativo, descriptivo, no experimental, con diseño transaccional.

\section{Muestra}

A través de muestreo probabilístico de tipo no intencional se seleccionó 87 docentes universitarios de la ciudad de Sincelejo, sin embargo, se permitió la participación de 83 docentes quienes cumplieron con los criterios de inclusión: Trabajar en una institución de educación en un horario superior 8 horas diarias, 40 horas semanales, con mínimo 10 horas de clase, y trabajo remoto en casa. Como criterio de exclusión: Tener algún tipo de discapacidad, diagnosticados en el último año con enfermedad laboral o accidente de trabajo, docentes irregulares y que no firmaran el consentimiento para su participación.

\section{Instrumentos}

Para la toma de datos se aplicó una entrevista estandarizada para obtener información sociodemográfica, antecedentes personales y ocupación. Además, se empleó el cuestionario estandarizado denominado Nórdico de Kourinka publicado en $1987^{(16)}$, validado y usado en diferentes países para el tamizaje de EME desde un contexto ergonómico y biomecánico(17), aclarando que no se recomienda como diagnóstico clínico(18).

Este cuestionario consiste en una serie de preguntas de opción múltiple que mide la ocurrencia de síntomas osteomusculares en las principales regiones anatómicas de las cuales se tomaron para esta publicación los resultados de presencia de dolor en los ítems que corresponden a muñeca- mano, codoantebrazo, hombro. La encuesta se envió a través de correo electrónico (e-mail) y vía WhatsApp, para el diligenciamiento por medio del aplicativo $\mathrm{y}$ almacenamiento en red GoogleDocs ${ }^{\circledR}$.

Para establecer bases de validación y confiabilidad del instrumento se consultó la teoría atendiendo la aprobación en distintos países de habla hispana, en este sentido México reporta en su estudio de validación una confiabilidad muy buena con un Alpha entre 0,855 y 0,860(19). Asimismo, los autores también reportan datos de otras investigaciones exponiendo resultados de validez positivos en la versión de España y Perú( 9$)$, siendo esta última validada a juicio de expertos ${ }^{(19) .}$

De esta misma forma, para la validación interna en el contexto aplicado, se contó con el criterio de tres expertos en el área, se aplicó posteriormente una prueba piloto a 56 docentes para verificar la confiabilidad y coherencia se empleó la prueba estadística Alpha de Cronbach, arrojando una puntuación de 0,736, de esta forma, teniendo en cuenta que como criterio de evaluación el valor debería ser superior a 0,7 , se concluyó que el 
instrumento de medición es adecuado en cuanto a su consistencia interna, obteniendo resultados confiables para su aplicación.

Además, se utilizó como soporte la herramienta validada propuesta por el Ministerio de Salud y Protección Social colombiano, denominada Batería de Riesgo Psicosocial(16), que contiene también elementos del modelo dinámico de Villalobos según lo confirmado por Uribe-Prado(20), pero sólo se reportan para este registro los datos del nivel intralaborales.

\section{Análisis estadístico}

Para el análisis de los datos se utilizó software estadístico SPSS versión 25, se procesaron las variables cuantitativas a través del análisis de mínimo, máximo, media y desviación estándar con tablas de frecuencia para las variables relacionadas con sintomatología osteomuscular y riesgo psicosocial intralaborales, así como sexo y nivel de actividad física. Para establecer prueba de normalidad se utilizó el estadístico Kolmogorov Smirnov con la corrección de significación de Lilliefors, cuyo resultado presentó un nivel de significancia igual a 0,000 ; en consecuencia, se rechazó la hipótesis de normalidad. Consecutivamente se utilizó la prueba de asociación no paramétrica $\mathrm{Chi}^{2}$ para definir el nivel de significancia estadística con un $p<0,05$.

\section{Consideraciones éticas}

Este proceso evaluativo se considera de bajo riesgo, atendiendo al artículo 11 contemplado en la Resolución 008430 de 1993 del Ministerio de Salud Colombiano, teniendo en cuenta, que se utilizaron pruebas no invasivas, estandarizadas y validadas científicamente por expertos temáticos, asimismo estas pruebas no infringieron con la integridad física, moral y social de los participantes evaluados en el estudio(21,22). Contó con el aval de la Corporación Universitaria del Caribe CECAR, acta 004 de 2021.
Asimismo, los partícipes firmaron consentimiento informado para la intervención en el estudio.

\section{Resultados}

En la Tabla 1 se presentan valores descriptivos cuantitativos de las variables consideradas para este estudio de relevancia a nivel sociodemográfico, describiendo así la población de estudio, adulta con edades entre 34 y 61 años de edad, el 71,1\% de sexo femenino, con un promedio de 8 años de desempeño en el puesto de trabajo, lo que denota el tiempo de vinculación y un promedio de estrato clasificado como 3 , lo que para el contexto colombiano se refiere a un nivel socioeconómico medio alto. En cuanto al nivel de homogeneidad, se encontró una significancia estadística entre la edad y los grupos con y sin dolor músculo esquelético.

En la Tabla 2 se evidencia las frecuencias estadísticas con respecto a la variable síntoma osteomuscular con y sin dolor, y zonificados por regiones corporales, atendiendo a esto, se reporta un patrón de presencia de dolor del 85,5\%, y las áreas comprometidas son el cuello con un $81,9 \%$, la espalda dorsal o lumbar con $72,3 \%$ y con un $45,8 \%$ presencia del dolor en muñeca o mano, con lateralidad derecha atendiendo a la dominancia.

En cuanto a la descripción estadística de los factores de riesgo psicosocial intralaborales se encontró con mayor prevalencia el dominio Liderazgo y relaciones Sociales con un 75,9\%, seguido del dominio Recompensa con un 61,5\%, luego el dominio Control en el trabajo con un 60,3\% y el dominio Demandas del trabajo con un 59\%. Estos resultados emergen de la suma de los indicadores reportados como alto y muy alto.

En cuanto a los niveles de actividad física, se reportan niveles considerados como bajo o inactivo con un $62,7 \%$.

Tabla 1. Variables sociodemográficas, pruebas de homogeneidad con el dolor músculo esquelético

\begin{tabular}{|c|c|c|c|c|c|c|}
\hline Variable & $\mathbf{N}$ & Mínimo & Máximo & Media & Desv. & $\mathbf{U}$ \\
\hline Edad & 83 & 34,00 & 61,00 & 44,9 & 8,4 & 0,02 \\
\hline Años de experiencia en el puesto de trabajo & 83 & 1,00 & 21,00 & 8,2 & 5,92614 & 0,19 \\
\hline Estrato socio económico de la vivienda & 83 & 2,00 & 4,00 & 3 & 64779 & 0,91 \\
\hline
\end{tabular}

U: U de Mann-Whitney; (p>0,05)

Desv: Desviación 
Tabla 2. Sintomatología osteomuscular, riesgos psicosociales intralaborales y nivel de actividad física

\begin{tabular}{|c|c|c|c|}
\hline Variables & & Frecuencia & (\%) \\
\hline \multirow[t]{2}{*}{ Sexo } & Femenino & 59 & 71,1 \\
\hline & Masculino & 24 & 28,9 \\
\hline Presencia de & $\mathrm{Si}$ & 71 & 85,5 \\
\hline $\begin{array}{l}\text { Dolor músculo } \\
\text { esquelético }\end{array}$ & No & 12 & 14,5 \\
\hline \multirow[t]{2}{*}{ Dolor en cuello } & $\mathrm{Si}$ & 68 & 81,9 \\
\hline & No & 15 & 18,1 \\
\hline \multirow{2}{*}{$\begin{array}{l}\text { Dolor dorsal o } \\
\text { lumbar }\end{array}$} & $\mathrm{Si}$ & 60 & 81,9 \\
\hline & No & 23 & 27,7 \\
\hline \multirow{2}{*}{$\begin{array}{l}\text { Dolor en } \\
\text { hombros }\end{array}$} & $\mathrm{Si}$ & 6 & 7,2 \\
\hline & No & 77 & 92,8 \\
\hline \multirow{2}{*}{$\begin{array}{l}\text { Lateralidad del } \\
\text { dolor en } \\
\text { hombros }\end{array}$} & $\begin{array}{l}\text { No hay } \\
\text { dolor }\end{array}$ & 77 & 92,8 \\
\hline & Derecha & 6 & 7,2 \\
\hline \multirow{2}{*}{$\begin{array}{l}\text { Dolor en codo o } \\
\text { antebrazo }\end{array}$} & $\mathrm{Si}$ & 3 & 3,6 \\
\hline & No & 80 & 96,4 \\
\hline \multirow{2}{*}{$\begin{array}{l}\text { Lateralidad del } \\
\text { dolor en codo o } \\
\text { antebrazo }\end{array}$} & $\begin{array}{l}\text { No hay } \\
\text { dolor }\end{array}$ & 80 & 96,4 \\
\hline & Derecha & 3 & 3,6 \\
\hline \multirow{2}{*}{$\begin{array}{l}\text { Dolor en muñeca } \\
\text { o mano }\end{array}$} & $\mathrm{Si}$ & 38 & 45,8 \\
\hline & No & 45 & 54,2 \\
\hline \multirow{3}{*}{$\begin{array}{l}\text { Lateralidad del } \\
\text { dolor en muñeca } \\
\text { o mano }\end{array}$} & $\begin{array}{l}\text { No hay } \\
\text { dolor }\end{array}$ & 45 & 54,2 \\
\hline & Derecha & 35 & 42,2 \\
\hline & Bilateral & 3 & 3,6 \\
\hline \multirow{5}{*}{$\begin{array}{l}\text { Demandas del } \\
\text { trabajo }\end{array}$} & Sin riesgo & 16 & 19,3 \\
\hline & Bajo & 14 & 16,9 \\
\hline & Medio & 4 & 4,8 \\
\hline & Alto & 30 & 36,1 \\
\hline & Muy alto & 19 & 22,9 \\
\hline \multirow{5}{*}{$\begin{array}{l}\text { Control en el } \\
\text { trabajo }\end{array}$} & Sin riesgo & 17 & 20,5 \\
\hline & Bajo & 14 & 16,9 \\
\hline & Medio & 2 & 2,4 \\
\hline & Alto & 33 & 39,8 \\
\hline & Muy alto & 17 & 20,5 \\
\hline \multirow{5}{*}{$\begin{array}{l}\text { Liderazgo y } \\
\text { relaciones } \\
\text { sociales }\end{array}$} & Sin riesgo & 7 & 8,4 \\
\hline & Bajo & 7 & 8,4 \\
\hline & Medio & 6 & 7,2 \\
\hline & Alto & 37 & 44,6 \\
\hline & Muy alto & 26 & 31,3 \\
\hline \multirow[t]{5}{*}{ Recompensas } & Sin riesgo & 12 & 14,5 \\
\hline & Bajo & 14 & 16,9 \\
\hline & Medio & 6 & 7,2 \\
\hline & Alto & 33 & 39,8 \\
\hline & Muy alto & 18 & 21,7 \\
\hline \multirow{3}{*}{$\begin{array}{l}\text { Nivel de } \\
\text { actividad física }\end{array}$} & Nivel alto & 17 & 20,5 \\
\hline & $\begin{array}{l}\text { Nivel } \\
\text { moderado }\end{array}$ & 14 & 16,9 \\
\hline & $\begin{array}{l}\text { Nivel bajo o } \\
\text { inactivo }\end{array}$ & 52 & 62,7 \\
\hline
\end{tabular}

Al comparar la variable dolor músculo esquelético con los factores de riesgo psicosocial a nivel intralaborales y el nivel de actividad física, se obtuvo una relación estadísticamente significativa entre las personas con y sin dolor y las variables dominio y control en el trabajo $(p>0,05)$; inherentes a los factores de riesgo psicosociales intralaborales (Tabla $3)$.

Tabla 3. Pruebas de asociación dolor músculo esquelético con riesgo psicosocial intralaborales y nivel de actividad física

\begin{tabular}{llrr}
\hline \multicolumn{1}{c}{ Dolor vs... } & \multicolumn{2}{c}{$\begin{array}{c}\text { Coeficiente de } \\
\text { asociación }\end{array}$} & $\begin{array}{c}\text { Sig. } \\
\text { (bilateral) }\end{array}$ \\
\hline $\begin{array}{l}\text { Demandas del trabajo } \\
\text { Control en el trabajo }\end{array}$ & Phi & 0,277 & 0,175 \\
Liderazgo y relaciones & Phi & 0,405 & 0,009 \\
sociales & & 0,128 & 0,852 \\
Recompensas & Phi & 0,217 & 0,419 \\
Nivel de actividad física & Phi & 3,04 & 0,218 \\
\hline *Phi: Chi-cuadrado; $(p>0,05)$ & & &
\end{tabular}

\section{Discusión}

En este estudio se buscó identificar la relación entre el dolor músculo esquelético y factores de riesgo psicosocial intralaborales presentes en el ambiente de trabajo de la práctica docente, para destacar la importancia que tiene la práctica regular de actividad física en el contexto laboral. De la misma forma, Carvajal(23) publica resultados similares, encontrándose la mayor prevalencia de dolor a nivel de la región lumbar, seguido del cuello y hombro, así también reporta a nivel de riesgo psicosocial un mayor porcentaje en los dominios: recompensa, liderazgo y relaciones social, datos que guardan un alto valor de coincidencia con el actual. Sin embargo, la población de estudio es del área de salud, profesionales que de una u otra forma tiene un rol de enseñanza ${ }^{(24) .}$

Asimismo, un estudio realizado a docentes del área de odontología que aplicó el cuestionario Nórdico para evidenciar la presencia de dolor musculo esquelético, arrojó resultados similares a los reportados en la actualidad, donde se descubrió que las áreas de mayor incidencia de dolor son la espalda dorsal o lumbar, el cuello, seguida de mano, mientras que la menor recurrencia estaba en antebrazo ${ }^{(22)}$.

También, el estudio denominado "Prevalencia de trastornos musculo esqueléticos en profesores universitarios que realizan teletrabajos en tiempos COVID-19 en Perú", se encontró que el total de la población estudiada tiene dolor a nivel de los segmentos reportados en el cuestionario nórdico Kuorinka, a diferencia de este estudio donde la prevalencia del dolor sólo se presentó en el 85,5\%. Además, se reporta que la distribución de dolor por 
segmentos en mayor porcentaje se presenta en columna dorso-lumbar con $67,27 \%$, cuello con $64,55 \%$ y menor porcentajes para hombro, muñeca, mano, codo y antebrazo ${ }^{(9)}$.

Este trabajo reporta algo similar, ya que se encontró los mayores porcentajes de reporte de dolor musculo esquelético a nivel de cuello y columna dorsal o lumbar y menor porcentaje de dolor en hombros, codo y antebrazo. A diferencia de los hallazgos de Mendoza et al.(2), quien evidenció que el dolor en hombros ocupa el primer lugar entre los síntomas más frecuentes en la población.

En cuanto a la variable riesgo psicosocial, esta se definen como las condiciones presentes en el contexto laboral(25), dentro del entorno físico, el ambiente social(26), contenido del trabajo y la organización misma, además, tiene capacidad para afectar tanto el bienestar(27) o la salud física del trabajador, así como se afecta el desarrollo del trabajo(28), lo que puede llegar a convertirse en un trasmisor de salud o de enfermedad para el trabajador(29).

De esta forma, la descripción estadística de los factores de riesgo psicosocial intralaborales estudiados alcanzó una mayor prevalencia de riesgo en los dominios Liderazgo - Relaciones Sociales y Recompensa, resultados similares a los obtenidos por Carvajal Vera, quien plantea que puede haber un papel de predisposición somatizante como posible predictor de EME, lo cual puede actuar como un intercesor concluyente en la respuesta específica e individualizada a las exposiciones desencadenantes, como el aumento de la actividad en el trabajo(23).

Asimismo, las asociaciones significativas encontradas entre las variables de presencia de molestia o dolor musculo esquelético, y riesgo psicosocial intralaborales para este estudio es el dominio control en el trabajo $(p>0,05)$, variables que explican que el mayor riesgo depende de las percepciones de los trabajadores en cuanto a los requerimientos que el oficio atribuye al sujeto, las cuales pueden llegar a ser de distinto origen, como las cuantificables, cualitativas, emocionales, de responsabilidad, del ambiente físico, laboral y de la jornada de trabajo; que a su vez pueden llegar a influir sobre distintos aspectos que interceden en la actuación laboral(18).

A diferencia de otros estudios donde la asociación se realizó de acuerdo al segmento alterado con el síntoma de dolor y las significancias estadísticas, se dieron a nivel de la región anatómica afectada con dolor, asociado a los distintos dominios psicosociales(22).

\section{Conclusiones}

El primer síntoma presente ante la probabilidad de una alteración a nivel músculo esquelético es la presencia del dolor, condición que perturba el bienestar del trabajador, obligándolo a realizar trabajos bajo posturas protectoras inadecuadas y que con el tiempo pueden llegar a convertirse en enfermedades laborales, llegar a causar accidentes y convertirse en uno de los índices de mayor aumento de ausentismo a nivel laboral.

Sumando a esto, la presencia de dolor se conjuga de tal forma que interviene dentro de la condición psicosocial del individuo, alterando no sólo el desempeño laboral, sino también las condiciones de evaluación en la práctica disminuida a causa de las molestias que se generan, lo que se manifiesta en las conductas expuestas ante las exigencias emocionales del trabajo, reflejadas en cansancio y sensación de fatiga y dolor muscular.

Es por esto, que estudiar las distintas variables que intervienen a nivel laboral denota siempre un alto índice de relevancia, teniendo en cuenta, que las condiciones del trabajo conllevan dentro de su práctica una lista de factores que obligan a permanecer en constante actualización de su contenido y así de alguna manera llegar a mejorar los procesos de atención, intervención y dosificación de las cargas intra y extra laborales en busca de una armonía entre ellos, que se evidencie en una mejora de la condición de salud física, mental y social contribuyendo a la productividad ocupacional.

Conflicto de intereses: Ninguno declarado por los autores.

\section{Referencias}

1. Franco Chávez SA, Salazar Paramo M, Peña Ortiz MO, Aguilera Velasco $\mathrm{M}$ de los A. Enfermedades músculo esqueléticas por agentes ergonómicos en trabajadores afiliados al Instituto Mexicano del Seguro Social, México. Humanidades Médicas. 2017;6(1). DOI: 10.37467/gkarevmedica.v6.1466.

2. Mendoza Fritz ME, Lora Flórez B, Pérez Martínez J, Arrazola David MJ. Condiciones de salud asociados con la aparición de trastornos osteomuscular en docentes de la institución educativa despertar del sur en la ciudad de Barranquilla. 
Ingeniería, Desarrollo e Innovación. 2020;2(2). DOI: 10.32012/26195259/2020.v2i2.78.

3. López Laverde J. Prevalencia de sintomatología osteomuscular en miembro superior y fatiga laboral en una empresa de manufactura de la ciudad de Cali. Ergonomía, Investigación y Desarrollo. 2020;2(3):99-116. DOI: 10.29393/EID2-8PSJL10008.

4. Bedoya Marrugo EA, Osorio Giraldo IC, Tovar Henao C, Roqueme Suarez K, Espinosa Fuentes EA. Determinación de la carga física como factor de riesgo de desórdenes osteomusculares. Espacios. 2018;39(6):10. Disponible en: http://ww.revistaespacios.com/a18v39n06/18390610.htm l

5. Orozco-Vásquez MM, Zuluaga Ramírez YC, Pulido Bello G. Factores de riesgo psicosocial que afectan a los profesionales en enfermería. Revista Colombiana de Enfermería. 2019;18(1):e006. DOI: 10.18270/rce.v18i1.2308.

6. Castillo-Ante L, Ordoñez-Hernández C, Calvo-Soto A. Carga física, estrés y morbilidad sentida osteomuscular en trabajadores administrativos del sector público. Universidad y Salud. 2020;22(1):17-23. DOI: 10.22267/rus.202201.170.

7. Hill MW, Duncan MJ, Oxford SW, Kay AD, Price MJ. Effects of external loads on postural sway during quiet stance in adults aged 20-80 years. Applied Ergonomics. 2018;66:64-9. DOI: 10.1016/j.apergo.2017.08.007.

8. Ohlendorf D, Troebs P, Lenk A, Wanke E, Natrup J, Groneberg D. Postural sway, working years and BMI in healthy truck drivers: an observational study. BMJ Open. 2017;7(7). DOI: 10.1136/bmjopen-2016-013281.

9. García-Salirrosas EE, Sánchez-Poma RA. Prevalencia de trastornos musculoesqueléticos en docentes universitarios que realizan teletrabajo en tiempos de COVID-19. Anales de la Facultad de Medicina. 2020;81(3):301-7. Disponible en: http://www.scielo.org.pe/scielo.php?script=sci_arttext\&pid $=\mathrm{S} 1025-55832020000300301$

10. Sánchez Sánchez AM, Sánchez Sánchez FJ, Ruiz-Muñoz D. Riesgos laborales en las empresas de residuos sólidos en Andalucía: una perspectiva de género. Saúde e Sociedade. 2017;26(3):798-810. DOI: 10.1590/S010412902017162878 .

11. Organización Mundial de la Salud. Actividad física [online]. acceso 2021 Oct $10 . \quad$ Disponible en: https://www.who.int/es/news-room/factsheets/detail/physical-activity

12. Casimiro Andújar J, Rodríguez Pérez A. Actividad física y salud en la empresa. En: Padilla Góngora D, Aguilar Parra JM, López Liria R, editores. Salud y ciclo vital. Madrid: Dykinson; 2019. p. 105-16. Disponible en: https://www.torrossa.com/en/resources/an/4494750

13. Sultan S, Abdel-Malek K, Arora J, Bhatt R. Human Simulation System for Injury Assessment Due to Repetitive Loading. En: Cassenti C, editor. Advances in Human Factors in Simulation and Modeling. Vol 591. 1ra ed. Los Ángeles: Springer, Cham; 2017. p. 131-40. DOI: 10.1007/978-3-319-60591-3_12.

14. Gönen D, Karaoğlan AD, Ocaktan MAB, Oral A, Atici H, Kaya B. Kas iskelet sistemi rahatsızlıklarının analizinde yeni bir risk değerlendirme yaklaşımı. Faculty of Engineering and Architecture of Gazi Universit. 2018;33(2):425-40. DOI: 10.17341/gazimmfd.416351.

15. Jiménez L, Lorence $B$, Hidalgo V, Menéndez S. Análisis factorial de las escalas FACES (Family Adaptability and Cohesion Evaluation Scales) con familias en situación de riesgo psicosocial. Universitas Psychologica. 2017;16(2). DOI: $10.11144 /$ Javeriana.upsy16-2.afef.
16. Albarrán V, Geldres V, Paredes P, Ramírez O, Ruiz F, Palomino JC. Validación de la batería de instrumentos para la evaluación de factores de riesgo psicosocial. Horizonte Médico (Lima). 2018;18(1):59-67. DOI: 10.24265/horizmed.2018.v18n1.09.

17. Cristina Acosta A, Jimenez Ruiz LK, Redondo Marin M, Pulido Guerrero EG. Estrés ocupacional y evaluación de desempeño en docentes universitarios del departamento del Cesar, Colombia. Encuentros. 2019;17(1):24-33. DOI: 10.15665/encuent.v17i01.1595.

18. González Muñoz EL. Estudio de validez y confiabilidad del cuestionario nórdico estandarizado, para detección de síntomas musculoesqueléticos en población mexicana. Ergonomía, Investigación y Desarrollo. 2021;3(1):8-17. Disponible en: http://revistas.udec.cl/index.php/Ergonomia_Investigacion /article/view/4339

19. Manchi Zuloeta FR. Posturas de trabajo y aparición temprana de síntomas músculo esqueléticos en estudiantes de odontología. Tesis de Grado. Lima: Universidad Nacional Mayor de San Marcos, Facultad de Odontología. 2017. Disponible en: https://hdl.handle.net/20.500.12672/6396

20. Uribe-Prado JF. Riesgos psicosociales, burnout y factores psicosomáticos en trabajadores del sector público. Investigación administrativa. 2020;49(125). DOI: 10.35426/iav49n125.03.

21. Gaviria Marulanda A, Osorio Ordoñez CC, Henao Morales M, Lenis Villada LF, Recalde Ruiz NA. Peligro biomecánico en la manipulación manual de carga en trabajadores de un ingenio azucarero. Revista Colombiana de Salud Ocupacional. 2021;11(2):e-6553. DOI: 10.18041/2322$634 \mathrm{X} / \mathrm{rcso} .2 .2021 .6361$.

22. Gaitán González LC. Aplicación del cuestionario nórdico de kuorinka a estudiantes y docentes odontólogos del área clínica y administrativa de la facultad de odontología de la universidad el bosque para identificar sintomatología dolorosa asociada a desórdenes musculoesqueléticos. Trabajo de grado. Bogotá D.C.: Universidad del Bosque; 2018. Disponible en: http://hdl.handle.net/20.500.12495/2440

23. Carvajal-Vera C, Aranda-Beltrán C, González-Muñoz E, LeónCortés S, González-Baltazar R. Desórdenes músculo esqueléticos y factores de riesgo psicosocial en el personal de enfermería de cuidados intensivos en Ecuador. MédicoCientífica de la Secretaría de Salud Jalisco. 2019;6(1):55-63. Disponible https://www.medigraphic.com/pdfs/saljalisco/sj2019/sj191h.pdf

24. Korhan O, Memon AA. Introductory Chapter: Work-related Musculoskeletal Disorders. 2019. p. 3-4. Disponible en: https://books.google.es/books?hl=es\&lr=\&id=zJj8DwAAQB AJ\&oi=fnd\&pg=PA3\&dq=Work-

related+Musculoskeletal+Disorders. $\% 3 B+2019 \&$ ots $=9 \mathrm{wdn}$ afrSCU\&sig=fAdejlf62cDB-

aV3DMkFxWQpBg8\#v=onepage \&q\&f=false

25. Ochoa Díaz CE, Centeno Maldonado PA, Hernández Ramos EL, Guamán Chacha KA, Castillo Vizuete JR. Occupational safety and health of workers and improvement of the work environment regarding active breaks. Universidad y Sociedad. 2020;12(5):308-13. Disponible en: https://rus.ucf.edu.cu/index.php/rus/article/view/1713

26. Toia Larsen M. Factores psicosociales y éstres laboral en trabajadores de las unidades de cuidados intensivos 
neonatales de dos Hospitales de Arequipa, Perú. SCIENTIARVM. 2019;5(1):29-37. Disponible en: http://scientiarvm.org/cache/archivos/PDF_021549763.pd $\mathrm{f}$

27. Vega Pérez JG, Vargas Ramos MF, Amores Guevara P del R, Arias Tapia SA. Riesgos psicosociales y la seguridad industrial en las lavanderías textiles del Cantón Pelileo. Sociedad Española de Estudios de la Comunicación Iberoamericana (SEECI). 2017;21(43):135-49. DOI: 10.15198/seeci.2017.43.135-149.
28. Salamanca Velandia SR, Pérez Torres JM, Infante Alvarado AF, Olarte Ardila YY. Análisis de los factores de riesgo psicosocial a nivel nacional e internacional. Temas. 2019;III(13):39-45. Disponible en: https://dialnet.unirioja.es/servlet/articulo?codigo=716907 1

29. Rivera-Porras D. Gestión del riesgo psicosocial y organizacional, un análisis bibliométrico. Aibi Revista de investigación, administración e ingeniería. 2019;7(1):26-30. DOI: $10.15649 / 2346030 X .49$. 